\title{
The management of diabetes in pregnancy
}

\author{
David R Hadden
}

\section{Summary}

The aim of the diabetes specialist is to provide a service to the pregnant diabetic woman so that she will present to her obstetrician with such well-controlled plasma glucose levels that her pregnancy will proceed without any diabetesrelated problem, and she will be delivered of a normal baby, of normal size, at the normal fullterm gestation, by the normal route. There are some problems in achieving this aim.

The exact definition of hyperglycaemia in pregnancy is still a matter of dispute. Screening methods to identify the problem differ widely. Many centres have developed joint diabetes/antenatal clinics, but there are practical problems with such an approach. Pre-pregnancy counselling, and discussion of contraceptive measures is an important task for the diabetologist and requires up-todate knowledge.

Control of plasma glucose requires alteration of insulin doses as pregnancy proceeds. Mothers with retinal, renal or cardiac problems will need special care. The medical problems which develop, and the management of blood glucose during labour and delivery, mean that the diabetes team must be very adjacent to the obstetric service, and a centralised approach offers many advantages. The postpartum state, and the long-term outcome for both mother and baby, remain both an interest and a responsibility for the obstetric physician.

Keywords: diabetes, pregnancy, management

Royal Victoria Hospital and Royal Maternity Hospital, Belfast BT12 6BA, Northern Ireland, UK

DR Hadden

\section{Definition}

In northern European populations, where insulin-dependent diabetes is more prevalent, most cases of hyperglycaemia in pregnancy are of this type, and the diagnosis is not in doubt. In Mediterranean and Far Eastern populations, insulin-dependent diabetes in young women is much less common, but a considerable number will develop hyperglycaemia for the first time in pregnancy. This is usually an indicator of subsequent non-insulin-dependent diabetes in the mother, although she will probably be treated with insulin during the current pregnancy. In the US both populations co-exist.

The actual diagnostic levels of venous plasma glucose necessary to establish diabetes can be decided according to the World Health Organization (WHO) criteria $^{1}$ (table 1) or by the US National Diabetes Data Group criteria which are based on the O'Sullivan studies in Boston 40 years ago $^{2}$ (table 2). Although in practice there may seem little difference, neither set of criteria has actually been fully validated in terms of pregnancy outcome, and more up-to-date studies are in progress. Unfortunately, as the glucose load in the two systems is different and the screening protocols to indicate the need for the glucose tolerance test are not at all similar, there is no clear conversion from one system to the other. At present, each group must decide which system it favours and keep to it. Both systems have been updated in the past 10 years. ${ }^{3,4}$

It may be possible in the future to move to a diagnosis of hyperglycaemia recognised for the first time in pregnancy based on a fasting plasma glucose, or on a glycosylated haemoglobin measurement, which would be much simpler. This would not, at present, meet the definition of gestational diabetes as 'carbohydrate intolerance of varying severity with onset or first recognition during pregnancy', but would satisfy the WHO definition of diabetes as 'a state of chronic hyperglycaemia'.

\section{Screening}

There have been a number of screening protocols in use to identify mothers during pregnancy who belong to one or other of the above diagnostic categories. The most robust is a $50 \mathrm{~g}$ oral glucose load given to every pregnant woman, with a single one-hour venous plasma glucose result above $140 \mathrm{mg} / \mathrm{dl}$ $(7.8 \mathrm{mmol} / \mathrm{l})$ as the threshold, but this has not been fully evaluated using modern laboratory and bedside methods. It also involves a glucose load for a great number of normal women. In Europe a number of clinical criteria have been used, including a family history of diabetes, glycosuria in a second fasting specimen, maternal weight greater than $90 \mathrm{~kg}$, a previous baby over $4.5 \mathrm{~kg}$, or a previous fetal abnormality or unexplained stillbirth. This limits the number of tests, but is certainly not inclusive of all mothers at risk. An enquiry into the screening protocols in clinical use in a number of maternity hospitals around London showed great differences in criteria and procedures, ${ }^{5}$ which would

Table 1 Interpretation of venous plasma glucose levels in pregnancy, using the $75 \mathrm{~g}$ oral glucose tolerance test, based on the WHO recommendations ${ }^{1}$ adapted to the criteria of the European Association for the Study of Diabetes. ${ }^{3}$

\begin{tabular}{llll}
\hline & Normal & Impaired glucose tolerance & Diabetes \\
\hline Fasting & $<6.0$ & $6.0-7.9$ & $\geqslant 8.0 \mathrm{mmol} / \mathrm{l}$ \\
& or & and & and $/ \mathrm{or}$ \\
2 -hour & $<9.0$ & $9.0-10.9$ & $\geqslant 11.0 \mathrm{mmol} / \mathrm{l}$ \\
& & $(162 \mathrm{mg} / \mathrm{dl})$ & $(200 \mathrm{mg} / \mathrm{dl})$ \\
\hline
\end{tabular}


Table 2 Criteria for the $100 \mathrm{~g}$ oral glucose tolerance test in pregnancy. At least two of the four values should be exceeded ${ }^{4}$

\begin{tabular}{ll}
\hline Time & Glucose level $(\mathrm{mg} / \mathrm{dl})$ \\
\hline fasting & $95(5.3 \mathrm{mmol} / \mathrm{l})$ \\
1 hour & $180(10.0 \mathrm{mmol} / \mathrm{l})$ \\
2 hour & $155(8.6 \mathrm{mmol} / \mathrm{l})$ \\
3 hour & $140(7.8 \mathrm{mmol} / \mathrm{l})$ \\
\hline
\end{tabular}

\section{Screening for}

hyperglycaemia in pregnancy

If abnormal result for one stage, proceed to next line

Urine glucose: every visit $\downarrow$

Random plasma glucose : first booking and 28 weeks

$$
\downarrow
$$

Standard $75 \mathrm{~g}$ carbohydrate breakfast test

$\downarrow$

Diet advice - repeat breakfast test if necessary

$\downarrow$

$75 \mathrm{~g}$ oral glucose tolerance test

$$
\downarrow
$$

Insulin treatment $\downarrow$

Postpartum random plasma glucose (or $75 \mathrm{~g}$ oral glucose tolerance test at postnatal visit if ordered for patients who have stopped insulin after delivery only) support the viewpoint that searching for hyperglycaemia in pregnancy is largely a waste of time. Nonetheless, there is a major body of opinion that even mild hyperglycaemia in pregnancy is an important outcome determinant. ${ }^{6}$

The screening protocol in use at the Royal Maternity Hospital, Belfast, uses a universal screen with urine glucose (nonspecific but easy) and a random, of untimed, venous plasma glucose both at first booking and at 28 weeks gestation $\stackrel{0}{\circ}$ (boxes $1 \& 2$ ). This blood test will certainly identify any unknown diabetic $\varrho$ mother who might otherwise be missed, but in Belfast this occurs only about 3 once in 1000 normal pregnancies. A meal test is more physiological than a $₫$ glucose bolus and we have identified the normal response to a standard $\stackrel{\complement}{\leftarrow}$ breakfast meal: only if this is exceeded is a $75 \mathrm{~g}$ oral glucose tolerance test $\overrightarrow{\overrightarrow{\vec{F}}}$ undertaken, and this is necessary only about 10 times a year in relation to over 3000 deliveries. But it must be remembered that hyperglycaemia occurring for $\frac{C}{O}$ the first time in pregnancy is a rare event in Belfast obstetric practice; in other $\overline{\bar{c}}$. countries, and in other ethnic groups, it is much more common. ${ }^{7}$ In such $\stackrel{\nabla}{\circ}$ centres, the clinicians must still choose between the routine $50 \mathrm{~g}$ one-hour

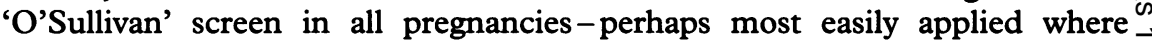
booking is late-or a selective system based on clinical criteria or random plasma glucose measurements to identify a subset of mothers who will receive $\overrightarrow{\vec{\omega}}$ testing.

\section{The combined antenatal/diabetes clinic: role of the diabetologist}

In the 'good old days' there was a recognition that pregnancy and childbirth was o at the heart of successful medical practice, and the family doctor tookresponsibility for all aspects of medical care. Now that specialisation has $N$ allowed major improvements in all parts of practice there is a danger that the internist may lose contact with a diabetic patient at crucial times, particularly during pregnancy. The concept of a combined antenatal and diabetes clinic, $s$ where the mother can be seen by the obstetric and the diabetic team has made it $\frac{\mathbb{O}}{0}$ possible for the diabetes physician to become involved in a meaningful way in $\stackrel{\mathbb{\Phi}}{\triangle}$ the progress of pregnancy. Similarly, close contact can be made with the neonatal specialists, though this is less often necessary.

The obstetrician's brief encounter with the mother gives a foreshortened and $\overrightarrow{0}$ incomplete view of the life-long disorder that is diabetes mellitus. Some tend to consider diabetes as a condition which complicates the nine months of pregnancy, whereas both the mother and the diabetologist are often more concerned about the long-term effects on the health of both mother and baby. The diabetologist's role during the pregnancy is to achieve such good diabetic control of the patient that she can present herself to the obstetrician in early $\stackrel{\square}{\mathbb{Q}}$ pregnancy requiring only routine antenatal observation and be delivered of a $\overrightarrow{\vec{\rho}}$ normally formed, normal-sized baby by the vaginal route at the full term of 3 pregnancy. ${ }^{8}$ This ideal will only be achieved some of the time, and it will require meticulous attention to detail by both the mother and her attendants.

Some obstetricians, or obstetric physicians, will be interested enough and $\bar{\partial}$ competent to undertake the whole management of both pregnancy and blood glucose control during the antenatal period. ${ }^{9}$ They should remember firstly that $\frac{5}{3}$ good communication is still necessary with the diabetes physician, who will be wondering where the patient has disappeared to, and who will certainly need to know the pregnancy outcome (most standard maternity hospital letters are addressed to the general practitioner and ignore a diabetes clinic). The obstetric junior staff may not be so motivated, or so experienced in insulin dosage, and a $\frac{T}{0}$ system for diabetes consultation should be available to them on a 24-hour basis. A truly perfectionist approach to this professional interface may be that which o has evolved in Stockholm, where the neonatal paediatrician with an interest in N diabetes takes over control of the mother's blood glucose in the interests of $\mathbb{W}^{\mathrm{N}}$ good fetal medicine.

A combined antenatal/diabetes clinic has been held at the Royal Maternity Hospital, Belfast, for over 30 years, and during that time the perinatal mortality in insulin-dependent diabetic pregnancy has fallen from over $10 \%$ to less than $\stackrel{\oplus}{?}$ $1 \%$. But there is still potential danger from an over-confident obstetrician usurping the diabetes physician's role, or from the physician offering obstetrical $\frac{}{\oplus}$ advice without the skill in that art which comes from long experience. The danger of the late pregnancy transfer from a peripheral hospital of a difficult $\stackrel{\Phi}{\varrho}$ case is well recognised. ${ }^{10}$

\section{Pre-pregnancy counselling: contraception}

The prospective mother should know that successful pregnancy in a diabetic woman requires special care. Most young insulin-dependent diabetics will have 
Screening for

hyperglycaemia in pregnancy

- Urine test for glucose (and protein) at every antenatal visit. If glycosuria present, consider blood glucose at any time

- Random, untimed blood glucose: capillary - self-monitoring strip or micro-cuvette. Venous plasma laboratory glucose - at first booking, at any other time if desired, and at 28 weeks

- If blood glucose at any time is $>6.6 \mathrm{mmol} / \mathrm{l}$, to have standard breakfast meal test (fasting) $-40 \mathrm{~g}$ carbohydrate as normal breakfast ( 2 rounds toast, 2 Weetabix or equivalent)-laboratory venous plasma glucose at 0 and 2 hours

- If fasting plasma glucose at standard breakfast meal test is $>6.6 \mathrm{mmol} / \mathrm{l}$ and/or 2-hour plasma glucose $>8.0 \mathrm{mmol} / \mathrm{l}$, to have $75 \mathrm{~g}$ oral glucose tolerance test (OGTT). If 2-hour plasma glucose $>9.0 \mathrm{mmol} / \mathrm{l}$ the diagnosis of hyperglycaemia in pregnancy is made and treatment with insulin considered. (A full OGTT has venous plasma glucose at $0,30,60,90,120$ min; a minimal OGTT has only the fasting and 120-min samples)

- Treatment with insulin is usually necessary when random plasma glucose is persistently above $8.0 \mathrm{mmol} / \mathrm{l}$, when the breakfast tolerance test shows 2 -hour venous plasma glucose $>8.0 \mathrm{mmol} / \mathrm{l}$, and $/$ or when the $75 \mathrm{~g}$ OGTT shows a 2-hour plasma glucose $>9.0$ $\mathrm{mmol} /$. Prior to starting insulin, assessment by a dietitian and advice on sensible eating patterns is necessary. If blood glucose is above $11.0 \mathrm{mmol} / \mathrm{/}$ at any time, the diagnosis of diabetes is established and insulin must be seriously considered

- Mothers diagnosed as having hyperglycaemia in pregnancy may have subsequent insulin-dependent diabetes or non-insulin-dependent diabetes. Postpartum review is necessary and subsequent measurement of urine glucose and capillary blood glucose at 6 weeks. Non-pregnant criteria for diagnosis of diabetes mellitus would then apply

Box 2 some knowledge of this, although their elders may be less well informed. Unless special consideration is given to all diabetic women of childbearing age, it is easy for the ground rules of superior diabetic control to be diluted by personal convenience, and this consideration is required by all members of the diabetes care team, both at hospital clinic and at primary care consultations. A small leaflet on Diabetes and babies has been in use at the Royal Victoria Hospital for many years, and is freely available, although it is up to the doctor or nurse specialist to identify the appropriate time to introduce it.

The value of a 'pre-pregnancy clinic' was first demonstrated by Steele in Edinburgh 20 years ago. ${ }^{11}$ Mothers who attended the clinic showed a considerable benefit in all aspects of their pregnancy. But pregnancy is not always a carefully planned exercise from either the diabetic or other points of view, though rarely is it totally unanticipated: there is some reasonable doubt about the value of 'preaching to the converted', and the real challenge is to get advice to those who need it in an acceptable and understandable form. The very regimented protocol of pre-conception blood glucose control achieved in the former East Germany in the early 1980s certainly suggested that congenital malformations might be completely prevented ${ }^{12}$ but this degree of success has not been achieved elsewhere.

Poorly controlled diabetes may itself be a cause of infertility, perhaps from a degree of suppression of cyclical pituitary function due to the stress of undernutrition; some studies have shown elevated serum prolactin in this situation. Contraception for diabetics of both sexes is no different than for anyone else, apart from the desire that pregnancy should not occur when the mother's blood glucose is not well controlled. The present low-dose combined contraceptive pills are fully acceptable and do not have any appreciable effect on blood glucose levels. Intra-uterine devices have been found to be rather more prone to localised infection, but are still used in older patients. The sheath also continues to be used, although present day Government advertising tends to stress its anti-AIDS value rather than its use in family planning in many healthcare situations. Many couples will decide to have a sterilisation procedure when they consider their family to be complete.

\section{Control of plasma glucose}

Present day received wisdom on optimum control of plasma glucose in nonpregnant young diabetics suggests that the best option is the basal/bolus regimen, with a later evening basal injection of long-acting insulin and pre-meal boluses of short-acting insulin, all given with pen injection devices. This system offers flexibility and convenience and is widely used. In pregnancy it may have some particular advantage because of the changing insulin resistance that occurs on a physiological basis, which requires day-to-day adjustment of dose in most mothers. The same effect can be achieved using a subcutaneous insulin infusion pump, but these have proved too complex for routine use. The highest blood glucose in tightly controlled patients is usually post-breakfast, and it is important that mothers do not over-respond to this reading and increase the bolus doses too much: the post-breakfast peak is short lived and is not closely related to overall glucose control as shown by glycated haemoglobin $\left(\mathrm{HbA}_{\mathrm{Ic}}\right)$.

There is evidence that good control in pregnancy can be achieved by variable mixtures of short- and medium-acting insulin taken twice daily, ${ }^{13}$ although many mothers find that splitting the evening dose of medium-acting insulin so that half of it is taken at bedtime avoids the chance of nocturnal hypoglycaemia. For this reason the present fixed mixtures available in pen devices may not be sufficiently flexible.

Brittle diabetes is a clinical syndrome, not necessarily associated with any identifiable alteration in biochemical mechanisms or insulin resistance, but certainly more common in young women, only some of whom may have a personality problem. Management of these patients before and during pregnancy may be difficult, and rapid swings from hyperglycaemia to hypoglycaemia can be a challenge to both doctor and patient. That there may be problems other than purely metabolic at the root of this syndrome is suggested by the improved control these same patients achieve once their pregnancy is complete and their family status assured. The monograph on this topic $^{14}$ is essential reading for either obstetrician or physician who feels that their ability to devise a suitable insulin regimen is being sorely tested.

Noncompliance is not necessarily the same condition and, particularly for teenagers and young adults, who may be especially likely to have unexpected pregnancies, every effort must be made by all members of the diabetes team to keep in touch. An adolescent clinic on a Saturday morning, or in the evening after school, with the same doctor each time who has made contact with the 
paediatric clinic before transfer, is helpful. A dedicated nurse specialist who can keep in touch, if necessary by telephone, and clinic staff who are generally aware and concerned about the particular problems of adolescence and growing up, can sometimes be found in answer to a newspaper advertisement: the success of diabetes management in young people may depend on them.

Without question, self-monitoring of capillary blood glucose has made the $\stackrel{0}{0}$ greatest difference to achieving good control, ${ }^{15}$ but constant finger pricking is a bore to some people and a further breakthrough is needed to simplify this $\underset{Z}{z}$ procedure. There is a long-established dictum that compliance is the most $\stackrel{\AA}{\varrho}$ important predictor of pregnancy outcome. ${ }^{16}$

\section{Complications}

The long-term complications of diabetes of particular relevance to pregnancy $\frac{\bar{\sigma}}{\bar{m}}$ are retinal, renal and myocardial. In the past these were not recognised until $\widehat{\odot}$ severe organ damage had been sustained, and were felt to be major contraindications to pregnancy, largely on account of risk to the mother, although there was also a greatly increased fetal mortality. More recently, with? improved screening procedures, the threshold for identification of these $\overrightarrow{\vec{\omega}}$ problems has fallen, and very early evidence of retinopathy (using retinal $\stackrel{\omega}{\circ}$ photography), nephropathy (using urinary micro-albumin assay), and myocar-8 dial ischaemia (using noninvasive cardiac scanning) can be obtained. $\underline{3}$. Discussion of the risks of these conditions is therefore dependent on the point $\mathrm{N}$ in the natural history of the complication that coincides with the pregnancy.

Diabetic retinopathy sufficient to cause visual loss from intra-ocular haemorrhage, vascular proliferation, or even ischaemic maculopathy is still a N real risk in young diabetic women. It is certainly related to previous poor 0 control of blood glucose, and these problems should be identified and treated ophthalmologically before pregnancy. ${ }^{17}$ Early retinopathy, usually micro- $\vec{s}$ aneurysms seen on fundoscopy or on retinal photography is probably present $\frac{\mathbb{D}}{0}$ in more than half of pregnancies in women with insulin-dependent diabetes, $\frac{\mathbb{\mathbb { D }}}{\overrightarrow{\mathrm{d}}}$ but only in about $10 \%$ of those with non-insulin-dependent diabetes. ${ }^{18} \frac{3}{8}$ Retinopathy often worsens during pregnancy, especially in the more advanced $\stackrel{\mathbb{}}{-}$ cases: this may be due either to the physiological changes of pregnancy, or to $\overrightarrow{0}$ rapid improvement in blood glucose control. Many of these early pregnancyrelated changes regress in the first year postpartum, so that a conservative approach is reasonable. Routine ophthalmological examination in diabetic pregnancy is essential, however, at a frequency determined by the severity of the eye disease.

Overt diabetic nephropathy (urine total protein greater than $300 \mathrm{mg} / 24 \mathrm{~h} \stackrel{\mathrm{Q}}{\mathbb{Q}}$ before 20 weeks gestation) can occur in up to $10 \%$ of some series of diabetic $\overrightarrow{\vec{F}}$ pregnancies, and is relatively more common in the older non-insulin-dependent $\stackrel{\circ}{3}$ diabetic mother. It is closely associated with hypertension, both chronic and pregnancy-induced, and also related to significant neonatal morbidity with growth retardation. Microalbuminuria (urine albumin $30-300 \mu \mathrm{g} / \mathrm{l}$ in a single specimen) is also associated with increased risk of hypertension and $\underline{\underline{B}}$ prematurity, but this is a rather variable measurement and a single assay is not sufficient. ${ }^{19}$ The long-term outlook for both mother and child is more serious when maternal renal impairment is severe, although it does not appear $\bigcirc$ that pregnancy specifically accelerates the rate of decline of renal function. ${ }^{20}$ Renal transplantation can be followed by successful pregnancy.

Myocardial ischaemia presenting during pregnancy is fortunately rare, but is $\frac{D}{0}$ a real hazard for some older diabetic women with established complications for whom pregnancy is a deeply cherished ambition. A history of angina or previous $\mathcal{N}$ infarction must raise the issue of the advisability of pregnancy: current and $N$ future developments in the mangement of coronary heart disease may improve $N$ the present bad prognosis, but it must be remembered that diabetes removes the epidemiological advantage of the female sex to the risk of ischaemic heart disease and pregnancy places a further strain on the myocardium.

\section{Medical problems in the first trimester}

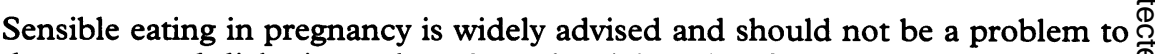
the concerned diabetic mother. A total weight gain of $10 \mathrm{~kg}$, with only $3 \mathrm{~kg}$ in $\stackrel{\mathbb{Q}}{\circ}$ the first trimester, would be reasonable. The best evidence of what pregnant $\overline{0}$ diabetic women actually eat comes from a study in Aberdeen, where, in spite of $\varnothing$ intense personalised diabetic advice, the current official recommendation to consume $50 \%$ energy from carbohydrate and less than $30 \%$ from fat was not achieved; snack foods, a sense of restriction, and local eating habits taking priority. ${ }^{21}$ Concern about an adequate intake of trace elements, folate, and free 
Sick days in pregnancy: diabetes advice

You are trying to keep your blood glucose as normal as possible all the time. If you have a day when you don't feel well, and don't feel like eating, what should you do?

The important thing is that you do still need your insulin, even though you are not eating your full diet plan.

- Always check your blood glucose at the usual times: also check urine for ketones

- Take your normal insulin dose at the normal times, even if you feel you can't eat anything

- Don't force yourself to eat solid foods: if possible, take your carbohydrate allowance as a drink. Even if you eat nothing the stress of sickness usually means that your blood glucose will not fall too low

- Do drink plenty of water or low calorie drinks

- If your blood glucose is high (above $10 \mathrm{mmol} / \mathrm{l}$ ) you should consider taking 4 units of short-acting insulin in addition to your usual dose, at breakfast, lunch, tea or suppertime. If it is above $20 \mathrm{mmol} / 1$ you should take 10 units of short-acting insulin and ask for medical advice

- If your blood glucose is low (below $2 \mathrm{mmol} / \mathrm{l}$ ) or you have a bad hypoglycaemic attack, take glucose in moderate quantity (not too much). Someone may need to give you a glucagon injection, so make sure that it is available. You will usually still need your insulin by the next mealtime, but you should consider reducing the dose by up to 4 units, and especially consider reducing the long-acting insulin if you take it

- Always get someone to help: the emergency advice number is -

\section{Box 3}

\section{Emergency home management of hypoglycaemia in early pregnancy}

- $10 \mathrm{~g}$ glucose as a proprietary tablet (Dextrosol ${ }^{\circledR}$ ), 2 sugar lumps, any $10 \mathrm{~g}$ carbohydrate exchange (apple, orange, glass of milk, slice of bread)

- $10 \mathrm{~g}$ glucose given as an intra-oral gel which can be administered to a patient who is becoming uncooperative (Hypostop ${ }^{\circledR}$ )

- $1 \mathrm{mg}$ glucagon by subcutaneous injection (the glucagon vial contains a powder and lasts for a long time until mixed with the water for injection immediately prior to administration: suitable instruction in this procedure is needed before the emergency occurs); follow by small snack radical scavengers to reduce the chance of fetal congenital malformations has some basis in animal experimental work, but has been difficult to confirm in clinical practice. Diabetic women on average have a rather better balanced dietary intake than non-diabetic women.

Hypoglycaemia in the first trimester is the most important problem: it may be the first evidence of pregnancy having occurred, but it may have serious consequences for the mother and needs considerable discussion. The physiological state of 'accelerated starvation' accounts for the rapid swings from hypo- to hyper-glycaemia, but there is also less awareness of impending hypoglycaemia, perhaps due to a pregnancy-specific diminished sympathetic reaction. These normal adaptations to the pregnant state are superimposed on the overall desire to keep blood glucose normal, and it is not surprising that symptomatic hypoglycaemia becomes a real problem, especially for those mothers who are doing their best to follow perceived medical advice.

Those who have previously been excellently controlled on twice-daily shortand medium-acting insulins become prone to hypoglycaemia before the evening meal or at about $04.00 \mathrm{~h}$. These tendencies are greatly lessened by splitting the evening dose of medium-acting insulin and taking half at $22.00 \mathrm{~h}$ or later. Those on tight control regimes with basal/bolus pen injectors may be helped by taking the basal long-acting insulin in the morning, or twice daily at half the dose, and generally by reducing the proportion of their total dose taken in the long-acting form. Much individual variation exists, but there does not seem to be a different response to animal or genetically engineered human insulins in pregnancy.

Severe hyperglycaemia and ketoacidosis used to have disastrous consequences in pregnancy and were always fatal in pre-insulin days: with the overall improvement in control, due mainly to self-monitoring procedures, this has become less of a threat and some centres consider it less important than hypoglycaemia. It would still seem useful for a mother to know how to test her urine for ketones, and it is surprising how uncommon this knowledge is becoming, again because of the blood glucose monitoring strips. Perhaps a blood ketone strip will become part of the self-monitoring procedure in pregnancy; such strips have been used in the management of severe diabetic ketoacidosis, ${ }^{22}$ although they are not yet marketed in the UK. Proper management of 'sick days' at home will be helpful in prevention of severe ketoacidosis (box 3).

Time spent with both the mother and father in discussing the value of glycated haemoglobin levels as a measure of overall control will assist mutual understanding during the first trimester. Ideally, glycated haemoglobin should be measured pre-conception and at monthly intervals during pregnancy. This is also the time to instruct the husband or partner in the administration of glucagon for severe hypoglycaemia-a single 1-mg subcutaneous injection will release enough hepatic glycogen to raise the mother's blood glucose in a few minutes, so that she can take some normal food without acute medical intervention. A simple protocol for emergency home management of hypoglycaemia is shown in box 4 . Some mothers allow the threat of hypoglycaemia to become an excuse for over-indulgence in chocolates, sweets, etc, which they then blame on the insulin; a reduction in dose may be needed.

\section{Second and third trimester}

After about 16 weeks gestation the increasing insulin resistance of normal pregnancy overcomes the tendency to sudden hypoglycaemic swings, but there is usually a need to increase the overall insulin dose to maintain normal blood glucose levels. In general, a woman with well-controlled diabetes can expect to almost double her pre-pregnancy insulin dose by 30 weeks gestation; she should be reassured that this does not mean that her diabetes has become worse, and that the dose will return to the usual level immediately the baby is born. (It is worthwhile recording the pre-pregnancy dose in the obstetric record as it is sometimes difficult to remember it nine months later, and there is a tendency for early discharge after delivery without waiting for the niceties of diabetic control.)

At joint diabetes/antenatal clinics, review can be every two weeks, but if all is proceeding satisfactorily a degree of shared care, either with obstetrical colleagues in a peripheral maternity unit or a well-organised general practice is possible. Mothers with either diabetic or obstetric complications should not be seen on a shared care protocol and special arrangements may be necessary to facilitate their attendance at the central diabetes/pregnancy clinic. ${ }^{10}$ Retinal laser photocoagulation may be necessary, and close contact with the ophthalmic clinic will facilitate the mother's attendance. Hypertension in pregnancy is likely to be the most important management problem and discussion between physician and obstetrician is important in the choice of drugs. Agents with 


\section{Management of diabetes in labour: a labour ward guide}

Blood glucose should be $3-6 \mathrm{mmol} / \mathrm{l}$ before and during delivery. Ensure that self-monitoring meters are accurately calibrated, and if in doubt get a laboratory check.

\section{Spontaneous labour}

It is not necessary for the mother to take her full regular diet and insulin dose while in labour. Light food and fluids are prescribed: maintain capillary blood glucose $3-6 \mathrm{mmol} /$, hourly measurement. Usually a $5 \%$ dextrose infusion is erected in the labour ward and the insulin dose adjusted as below: this is kept running until the mother is able to eat light meals and return to her pre-pregnancy insulin regimen.

\section{Induction of labour}

Either by oxytocin infusion or prostaglandin pessary in early morning. Give usual doses of insulin the day before but omit the late evening long-acting insulin for those on four daily injections. No morning breakfast or insulin in antenatal ward. Measure capillary blood glucose hourly from $06.00 \mathrm{~h}$.

In labour ward, $5 \%$ dextrose infusion with insulin (table 3). A light breakfast may be given as well if trial of labour is unsuccessful.

\section{Elective caesarean section}

Give usual doses of insulin the day before, but omit any late evening longacting insulin. No morning breakfast or insulin in antenatal ward. Measure capillary blood glucose hourly from $06.00 \mathrm{~h}$.

In labour ward $5 \%$ dextrose infusion with insulin throughout procedure (table 3).

\section{Postpartum}

On first day give approximately half of normal pre-pregnancy dose and light meals. Mother's blood glucose in range $6-8 \mathrm{mmol} / 1$ before meals.

\section{Breast feeding}

Dietitian to arrange to increase mother's diet. Insulin dose not to be increased and may need to be decreased. Mother's food always before breast feeding to avoid risk of maternal hypoglycaemia if baby is slow to feed.

\section{Box 5}

proven safety during embryogenesis (methyldopa, hydralazine) should be used in early pregnancy, and other agents such as calcium channel blockers and alpha-1-receptor antagonists may be added in later stages. Beta-receptor blockers may increase the risk of serious hypoglycaemia and should be usedo with caution. Angiotensin-converting enzyme (ACE) inhibitors should never been used in pregnancy because of the potential for adverse effects on fetal rena? development and function; this is important to remember, as these drugs are ${ }^{\varrho}$ now quite widely used in early diabetic microalbuminuria, and great care is needed to avoid conception while taking an ACE inhibitor. ${ }^{23}$

\section{Labour and delivery}

The diabetes staff should make sure that they are known to the labour wardo midwives as the routine management of fluid and drug therapy is under theirs supervision. A protocol for insulin dosage, adjusted for any particular customs $\mathbb{D}$ peculiar to the hospital, should be prepared and agreed by diabetologist obstetrician, anaesthetist, neonatologist and, most importantly, the labour wardsister-in-charge. It is not always easy to get these people together and they mayhave particular views, for example, on how intravenous fluids are given orw recorded, and how insulin or other drugs are added to infusions. It is alsoo necessary for the diabetes team to be aware of the procedures used in spontaneous labour, for induction of labour, and for both emergency and elective caesarean section. The protocol in use at the Royal Maternity Hospitals Belfast, is shown in box 5 .

The diabetes team should be able to provide an on-call service for the maternity hospital so that immediate decisions about insulin dosage and intravenous or oral feeding can be facilitated. If the maternity hospital is isolated, or there is no regular diabetes/antenatal clinic, this degree of cooperation is very difficult to achieve.

Insulin can be given during labour by the usual subcutaneous route if al尺 proceeds rapidly. Most often, good control during labour can be achieved by adding a small amount of insulin (6 units) to a slow intravenous infusion of $500 \mathrm{ml} \mathrm{5 \%}$ dextrose, which will allow that amount of glucose to be metabolised without risk of hypoglycaemia (table 3). If more insulin is needed because of: hyperglycaemia it can be given subcutaneously or intravenously, depending on the speed of action needed, but it is best not to speed up the existing infusion too give more insulin because of the risk of fluid overload. If hypoglycaemia is as problem, $10 \%$ dextrose may be given for a while, but it is not necessary to giveo this hypertonic solution for long periods. Any other infusions, eg oxytocin or anaesthetic agents, must be taken into account; if necessary, all the insulin can ${ }^{\circ}$ be given separately by intramuscular injection or an intravenous syringe pump, $\overrightarrow{\vec{O}}$ but this usually only adds confusion at an already busy time.

\section{Postpartum}

Breast feeding is good clinical practice and should be encouraged. It does not make diabetes more difficult to control, and there is some epidemiological 3 . evidence that it may reduce the (small) chance of the baby itself developing insulin-dependent diabetes. From the diabetic nutritional viewpoint, breast milk contains lactose, and over 24 hours a lactating mother will lose about 50 go carbohydrate through her milk: this should be replaced by extra carbohydrate in the diet throughout the day, with some taken prior to breast feeds. It is important to avoid the chance of maternal hypoglycaemia while breast feeding, particularly if the baby is a slow feeder: the dose of insulin after delivery should ${ }^{\circ}$ always be reduced, and during breast feeding will probably be even less than theo normal non-pregnant dose. It is wise to aim for slightly higher preprandial $\vec{\omega}$ blood glucose levels, $6-8 \mathrm{mmol} / \mathrm{l}$, at this time, for the same reason. When the? time comes to wean to solid foods the mother must remember to reduce here own intake to avoid becoming fat.

\section{Outcome}

Always find out about the baby, even if it is temporarily in the nursery. A $\frac{\text { }}{\oplus}$ healthy mother and a healthy and thriving baby is the aim. The St Vincent $\frac{\rho}{\sigma}$ Declaration of the European Association for the Study of Diabetes and the World Health Organization aims for a pregnancy outcome at least as good as foro the non-diabetic mother. This goal has almost been achieved, due to as combination of factors, including obstetric and midwifery practice, neonatalo intensive care and intensive diabetic supervision with frequent maternal selfmonitoring of blood glucose. However, this intensive effort does not guarantee 
Table 3 5\% Dextrose and insulin guidelines in labour ward

\begin{tabular}{|c|c|c|c|c|c|}
\hline $\begin{array}{l}\text { Capillary blood glucose } \\
\text { (mmol/l) }\end{array}$ & $\begin{array}{l}\text { Infusion } 5 \% \text { dextrose } \\
(\mathrm{ml})\end{array}$ & $\begin{array}{l}\text { Insulin (short-acting) } \\
\text { added to each } 500 \mathrm{ml}\end{array}$ & $\begin{array}{l}\text { Time for } 500 \mathrm{ml} \\
\text { infusion (h) }\end{array}$ & Drops/min & $\begin{array}{l}\text { Supplementary subcuta- } \\
\text { neous short-acting insu- } \\
\text { lin every } 6 \text { hours }\end{array}$ \\
\hline$<2.0$ & 500 & 0 & 2 & 84 & 0 \\
\hline $2.0-3.9$ & 500 & 0 & 6 & 28 & 0 \\
\hline $4.0-7.9$ & 500 & $6 \mathrm{U}$ & 6 & 28 & 0 \\
\hline $8.0-11.9$ & 500 & $6 \mathrm{U}$ & 6 & 28 & $6 \mathrm{U}$ \\
\hline $12.0-15.9$ & 500 & $6 \mathrm{U}$ & 6 & 28 & $10 \mathrm{U}$ \\
\hline
\end{tabular}

1 World Health Organization Expert Committee on Diabetes Mellitus: Technical Report Series on Diabetes Mellitus: Tech

2 O'Sullivan JB, Mahan CM. Criteria for the oral glucose tolerance test in pregnancy. Diabetes 1964; 13: $278-85$.

3 Diabetes Pregnancy Study Group. A prospective multicentre study to determine the influence of pregnancy upon the $75 \mathrm{~g}$ oral glucose tolerance test (OGTT). In: Sutherland HW, Stowers JM, Pearson DWM, eds. Carbohydrate metabolism in pregnancy and the newborn, IV. London: Springer-Verlag, 1989; 209-26.

4 Carpenter MW, Coustan DR. Criteria for screening tests for gestational diabetes. $A m \mathcal{F}$ Obstet Gynecol 1982; 144: 768-76.

5 Nelson-Piercy C, Gale EAM. Do we know how Nelson-Piercy C, Gale EAM. Do we know how
to screen for gestational diabetes? Current to screen for gestational diabetes? Current practice in one Regional Heat
Diabetic Med 1994; 11: 493-8.

6 Langer O, Mazze R. The relationship between large-for-gestational-age infants and glycemic control in women with gestational diabetes. Am f Obstet Gynecol 1988; 159: 1478-83.

7 Hadden DR. Geographic, ethnic and racial variations in the incidence of gestational diabetes. Diabetes 1985; 34 (suppl 2): 8-12.

8 Hadden DR. Pregnancy problems in diabetics. In: Lawson DH, ed. Current medicine 2. Edinburgh: Churchill Livingstone, 1990; 87-104.

a normal outcome, and congenital malformations or hypertension-related complications still occur even in the best-regulated pregnancies. Large-fordates babies will not be abolished by maternal normoglycaemia, they will only return to the normal prevalence. If the mother's health is already seriously impaired by long-term diabetic complications the effect of a pregnancy may be deleterious.

The most optimistic aim in the future will be to abolish insulin-dependent diabetes altogether, and to identify and treat the early hyperglycaemia of noninsulin-dependent diabetes so that it ceases to be a pregnancy risk factor. Initial studies in both these fields are now in progress.

9 Gilmer MDG, Bickerton NJ. Advances in the management of diabetes in pregnancy: success through simplicity. In: Bonnar J, ed. Recent advances in obstetrics and gynaecology. Edinburgh: Churchill Livingstone, 1994; pp 51-78.

10 Traub AI, Harley JMG, Cooper TK, Maguines $S$, Hadden DR. Is centralized hospital care necessary for all insulin dependent pregnant diabetics? Br f Obstet Gynaecol 1987; 94: 957 62.

11 Steel JM, Johnston FD, Smith AF, Duncan LJP. Five years experience of a 'prepregnancy' clinic for insulin dependent diabetes. $B M \mathcal{F} 1982 ; 285$ : $353-6$.

12 Fuhrmann K, Reiher H, Semmler K, Fischer F, Fischer M, Glockner E. Prevention of congenital malformations in infants of insulin dependent diabetic mothers. Diabetes Care 1983; 6: 21923.

13 Small M, Cassidy L, Leiper JM, Paterson KR, Lunan CB, McCuish AC. Outcome of preg nancy in insulin dependent (type 1) diabetic women between 1971 and 1984. Qf Med 1986 61: $1159-69$.

14 Pickup JC, ed. Brittle diabetes. Oxford: Blackwell, 1985.

15 McCance DR, Hadden DR, Traub AI, Harley JMG. Self monitoring of capillary blood glucose in diabetic pregnancy. Pract Diabetes 1989; 6: $81-5$.
16 Pedersen J, Mølsted-Pedersen L. Prognosis of the outcome of pregnancy in diabetes: a new classification. Acta Endocrinol 1965; 50: 70-8.

17 Price JH, Hadden DR, Archer DB, Harley JMG Diabetic retinopathy in pregnancy. $\mathrm{Br} \mathcal{F}$ Obstet Gynaecol 1984; 91: 11-7.

18 Klein BEK, Moss SE, Klein R. Effect of pregnancy on progression of diabetic retinopathy. Diabetes Care 1990; 13: 34-40.

19 McCance DR, Traub AI, Harley JMG, Hadden DR, Kennedy I Urinary albumin excretion in diabetic pregnancy. Diabetologia 1989; 32: 236 -

20 Kimmerle R, Zass R-P, Cupisti S, et al. Pregnancies in women with diabetic nephropathy: long-term outcome for mother and child. thy: long-term outcome for moth

21 Anderson AS, Lean MEJ, Pearson DWM, Sutherland HW. A comparison between the diets of pregnant diabetic women and pregnan non-diabetic women. Diabetic Med 1990; 7: 452-6.

22 McBride MO, Smye M, Nesbitt GS, Hadden DR. Blood ketone monitoring with test strips and reflectance meter in diabetic ketoacidosis. Diabetic Med 1991; 8: 688-90.

23 Martin RA, Jones KI, Mendoza A, Barr M Benirschke $K$. Effect of ACE inhibition on the fetal kidney: decreased renal blood flow. Teratology 1992; 46: $317-21$. 